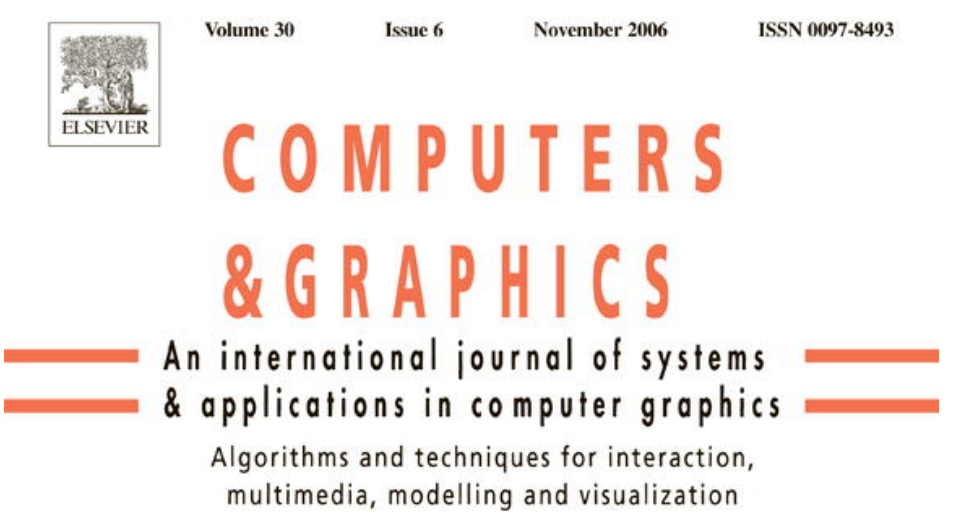

EOITOR-IN-CHIEF: J. L. Encarnação

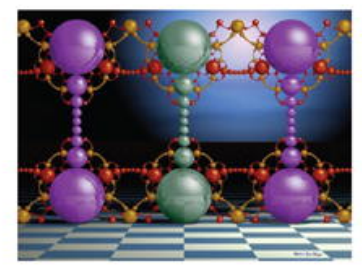

In this issue the special topic is MESH ANALYSIS
Guest Editors: Ayellet Tal, Thomas Funkhouser, Arik Shamir

This article was originally published in a journal published by Elsevier, and the attached copy is provided by Elsevier for the author's benefit and for the benefit of the author's institution, for non-commercial research and educational use including without limitation use in instruction at your institution, sending it to specific colleagues that you know, and providing a copy to your institution's administrator.

All other uses, reproduction and distribution, including without limitation commercial reprints, selling or licensing copies or access,

or posting on open internet sites, your personal or institution's website or repository, are prohibited. For exceptions, permission may be sought for such use through Elsevier's permissions site at:

http://www.elsevier.com/locate/permissionusematerial 


\author{
Technical Section
}

\title{
Simulation of atmospheric phenomena
}

\author{
Diego Gutierrez*, Francisco J. Seron, Adolfo Munoz, Oscar Anson \\ Department of Computer Science and Systems Engineering, University of Zaragoza, C/Maria de Luna 1, 50018 Zaragoza, Spain
}

Received 19 January 2006; accepted 12 May 2006

\begin{abstract}
This paper presents a physically based simulation of atmospheric phenomena. It takes into account the physics of non-homogeneous media in which the index of refraction varies continuously, creating curved light paths. As opposed to previous research on this area, we solve the physically based differential equation that describes the trajectory of light. We develop an accurate expression of the index of refraction in the atmosphere as a function of wavelength, based on real measured data. We also describe our atmosphere profile manager, which lets us mimic the initial conditions of real-world scenes for our simulations. The method is validated both visually (by comparing the images with the real pictures) and numerically (with the extensive literature from other areas of research such as optics or meteorology). The phenomena simulated include the inferior and superior mirages, the Fata Morgana, the Novaya-Zemlya, the Viking's end of the world, the distortions caused by heat waves and the green flash.
\end{abstract}

(C) 2006 Elsevier Ltd. All rights reserved.

Keywords: Atmospheric phenomena; Nonlinear ray tracing; Inhomogeneous media

\section{Introduction}

Simulating natural phenomena has always been one of the most challenging goals of computer graphics. In particular, the effect of the atmosphere on sunlight, under certain circumstances, causes a number of really spectacular phenomena; some are well-known, like the mirages, whereas some of them remain mostly unknown, such as the Fata Morgana or the Novaya-Zemlya. As far as the authors know some of the atmospheric phenomena presented in this paper have never been simulated before, including the last two mentioned above. This is due to the difficulty of first reproducing the exact, specific atmospheric conditions required to reproduce the effect and second, accurately calculating the nonlinear trajectories of light as it travels through the atmosphere under such conditions.

We present here our ray tracing method to reproduce some of these spectacular phenomena. The method is general and therefore can be applied to the simulation of any effect caused by nonlinear trajectories of light. We

\footnotetext{
*Corresponding author. Tel.: + 34976762354 ; fax: + 34976761914 .

E-mail addresses: diegog@unizar.es (D. Gutierrez), seron@unizar.es (F.J. Seron), adolfo@unizar.es (A. Munoz),oanson@unizar.es (O. Anson).
}

apply it here to simulate six different effects: the inferior and superior mirages, the Fata Morgana, the NovayaZemlya, the Viking's end of the world and the green flash, and propose several others, such as the distortions caused by heat waves or fire.

\subsection{Overview}

The atmosphere is a non-homogeneous medium, with its properties varying continuously from point to point. The most important property in terms of defining the light paths is the index of refraction, which in turn depends strongly on temperature, density and wavelength (and more subtly on other parameters such as humidity). The continuous variation of the light paths induced by the nonhomogeneous index of refraction can be described by a differential equation, as will be shown in Section 3.

Our simulation is based on three main points:

- Fermat's principle, which yields the physically based description of the light path through a non-homogeneous medium (with a continuously varying index of refraction), coupled with a fast, efficient numerical resolution method. 
- An accurate model of the atmosphere, which includes temperature and pressure at every point, as well as the equations that yield the index of refraction at every differential point at every wavelength, needed for the correct application of Fermat's principle.

- Our Atmospheric Profile Manager (APM), a tool to recreate the necessary atmospheric conditions, based on functions whose predicted values match actual measured data.

All the simulations presented in this paper are physically based. The equations are justified either by comparing the results with measured data or by citing previous works which have validated them. These references are mostly taken from other research areas such as optics or meteorology, as it is our conviction that accurate simulations of natural phenomena do require a multidisciplinary approach. Finally, although for this paper we focus on the Earth's atmosphere, our model is completely general and obviously valid for any medium.

The rest of the work is organized as follows: the next section describes the previous work and helps contextualize this research. Section 3 presents our simulation method, including a brief description of the physical laws involved, the model of the atmosphere, the equation describing the path of light and the resolution method. Section 5 validates the method, with rendered scenes shown in Section 4. Finally, Sections 6 and 7 discuss the conclusions and the future work.

\section{Previous work}

Modeling nature has always been one of the most ambitious goals of computer graphics. There are many phenomena that are caused by the varying non-homogeneous properties of the atmosphere, and consequently there has been a lot of work aimed at simulating them. These papers can be classified into two main categories:

Absorption and scattering related: These aim to obtain the color resulting from the interaction of light and the atmosphere. They consider that the atmosphere is a participating medium and therefore absorption and scattering events occur while light traverses it. Phenomena like clouds and rainbows are simulated this way.

Refraction related: These focus on the obtention of the true shape of what is seen. They consider that the atmosphere is a non-homogeneous media, and therefore light does not follow straight paths while traversing it. Phenomena like mirages and sunset effects can be simulated this way. This paper greatly contributes to this category.

\subsection{Absorption and scattering related}

Some of the simulations are focused towards the accurate obtention of color computation of the sky during daytime [1,2], nighttime [3] or even of the Earth's atmo- sphere as seen from the space [4]. These works are based on simplified models of the physics of the atmosphere, since they are mainly focused on performance rather than realism. Related to them we can find others which rely more on the physics of the atmosphere and therefore the color of the sky is more realistic and physically more accurate. For instance Irwin [5] takes into account Rayleigh scattering (due to the air molecules), and Jackèl and Walter [6] consider Mie scattering (due to the aerosols of the atmosphere). Mie scattering enables also cloud, rain and even rainbow simulations. In [7], by Riley et al., both Rayleigh and Mie scattering are considered, although many simplifications (like single scattering) are made in order to accelerate the render times by GPU. Dobashi et al. [8] also follow a similar approach. A very interesting survey about all the possible scattering related phenomena in the atmosphere is the work of Sloup [9], which presents different approaches and different models and phase functions for aerosols, ozone and clear air, and therefore sets the basis for the simulation of phenomena like clouds, halos or rainbows.

\subsection{Refraction related}

The first work considering the atmosphere as an inhomogeneous medium, in which the index of refraction varies at different heights, was developed by Berger et al. [10]. As a consequence, the light path is modified not only by the interaction with different objects, but by the own atmosphere as well. This way some effects like superior and inferior mirages can be simulated. Their model is based on a set of layers of the atmosphere at different heights with different indexes of refraction, and while tracing a light ray its path is refracted at the boundaries between two layers applying Snell's Law. On the other hand, Musgrave [11] rejects the previous model based on refraction and proposes a new one, based on total reflection. This model is also able to simulate some mirages, although it is rather incomplete.

The model for simulating the atmosphere by Lehn [12] is more complete. It is similar to the proposed in [10] but instead of pure refractions it considers parabolic paths of light inside the different layers, considering a set of perlayer individual properties. Linţu et al. [13] follow the work in [12], including also wavelength dependance, and consequently it is capable to simulate dispersion-based atmospheric phenomena, like the green flash. However, all these layer-based works are limited to one dimension variations of the index of refraction and are not capable of simulating other atmospheric effects like the distortion caused by fire.

A more general method is presented by Seron et al. [14]. It computes the path of light by calculating Fermat's principle using Euler's Method. This overcomes the limitations of previous works and therefore can simulate a broader range of phenomena, including mirages, some sunset effects or distortions caused by fire. 
This paper continues the work of [14], also solving Fermat's principle but improving it in the following ways:

- It is based not only on the laws of physics, but also on actual measured data borrowed from other areas of research. It includes a fully physically based characterization of the index of refraction of the atmosphere at every differential wavelength of the visible spectra and at every differential point of the volume, based on temperature and pressure profiles.

- It widens the range of phenomena which can be simulated (including effects never simulated before in computer graphics).

- It presents a much faster resolution method (orders of magnitude) which yields lesser errors.

- Being a spectral renderer (as opposed to single wavelength in [14]) it can simulate dispersion phenomena like the green flash, which could not be simulated before. Spectral data is converted to RGB prior to final display [15].

- It adds a way to intuitively characterize the temperature and pressure profiles atmosphere, based on the USA Standard Atmosphere, called the APM.

- It includes a model of the bleaching of photoreceptor cells in the human eye, used in the simulation of the green flash.

\section{The simulation process}

This section describes the underlying physics of our simulation, as well as the three main building blocks of the algorithm:

- An accurate description of the exact trajectory of light as it traverses the atmosphere, based on the laws of physics; the resulting differential equation will in turn need a robust, efficient numerical resolution method in order to keep rendering times within reasonable limits.

- A model of the atmosphere based on well-established standards, with a precise description of the index of refraction as a function of wavelength.

- A tool to de-standardize the atmosphere, in order to recreate the necessary conditions for the effects to occur. This has to be based on real data when available, borrowed from meteorological stations and scientific literature. For the functions used, resultant values must be compared with actual measured data.

\subsection{The trajectory of light}

When light passes from one homogeneous medium to another with a different index of refraction, its trajectory is modified according to Snell's law, which gives the relationship between angles of incidence and refraction, with the ray being continuous across the boundary:

$n_{1} \sin \theta_{i}=n_{2} \sin \theta_{t}$, where $n_{1}$ and $n_{2}$ are the indexes of refraction of the first and second medium respectively, $\theta_{i}$ is the incidence angle with respect to the normal to the boundary surface and $\theta_{t}$ is the angle of the refracted ray with respect to that normal. The index of refraction is defined as the quotient between the speed of light in that medium and the speed of light in a vacuum:

$n_{i}=\frac{v_{i}}{c}$.

This phenomenon is known as refraction, and it is traditionally accounted for in any ray tracer. It shows how light gets bent towards the medium with the greater index of refraction.

Snell's law is formulated considering two homogeneous media, through which light travels in a straight path. But all media are inhomogeneous at some degree, with their different properties varying from point to point, and the atmosphere of course is no exception. When the medium is inhomogeneous and the index of refraction changes continuously from point to point, the trajectory of light is affected at each differential step, not just at the boundaries between two media. The final result of this differential change is a curved path that distorts the normal view of a real scene, but that path cannot be calculated by traditional ray tracing. We apply Fermat's principle [16] to obtain the curved trajectory of the light rays. The original statement of Fermat's principle is "the actual path between two points taken by a beam of light is the one which is traversed in the least time." A reformulation of the principle can be expressed as "light, in going between two points, traverses the route $l$ having the smallest optical path length $\Lambda$ ". This optical path length $\Lambda$ is defined as the distance traveled in an optical system by light. For a series of continuous layers with index of refraction $n$ as a function of distance $l$, it is given by

$\Lambda=\int_{A}^{B} n_{\lambda} \mathrm{d} l$

where $n_{\lambda}$ is the index of refraction as a function of $l$ and $l$ is the distance (see Section 3.1 for further information). As a matter of fact, Snell's law is just a particular case of this principle [17]. In its differential form, it can be written as

$\mathrm{d} \Lambda=n_{\lambda} \mathrm{d} l$.

Under standard circumstances, the effects are negligible. It is under non-standard circumstances when we really see some amazing atmospheric phenomena. Probably the best known is the inferior mirage, which occurs when lower regions of the atmosphere are much hotter than higher regions, as in deserts. Since the index of refraction is an inverse function of temperature, light beams get bent upwards, and what we see on the ground is actually the image of objects above it. The effect is also known as the road mirage, since it is very usually seen in roads when the asphalt is much hotter than the surrounding air. Fig. 1 


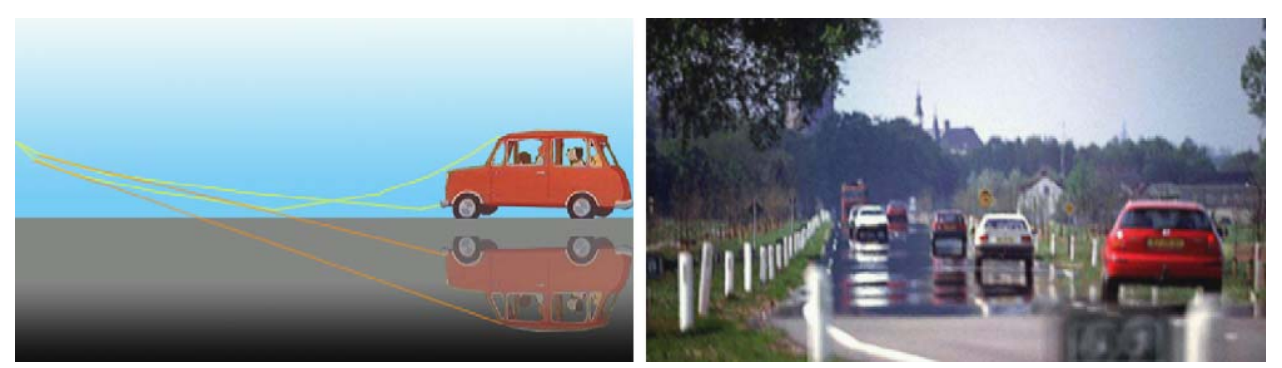

Fig. 1. Left: explanation of the origin of an inferior mirage. Right: picture of a real inferior mirage, also known as road mirage.

graphically explains this effect occurs, along with a real picture of the phenomenon.

Fermat's principle has been recently reformulated as "a light ray, in going between two points, must traverse an optical path length which is stationary with respect to variations of the path", as described by Glassner in [16]. The optical path length presents a stationary value whenever $\delta \Lambda=0$, therefore corresponding to a maximum, minimum or saddle point. Taking Eq. (3), $\delta \Lambda$ is given by

$$
\begin{aligned}
\delta \Lambda & =\delta \int_{A}^{B} n_{\lambda} \mathrm{d} l=\int_{A}^{B} \delta n_{\lambda} \mathrm{d} l+\int_{A}^{B} n_{\lambda} \delta(\mathrm{d} l) \\
& =\int_{A}^{B} \frac{\partial n_{\lambda}}{\partial x_{i}} \delta x_{i} \mathrm{~d} l+\int_{A}^{B} n_{\lambda} \delta(\mathrm{d} l),
\end{aligned}
$$

where $x_{i}$ are the components of $l$. Given that $\mathrm{d} l^{2}=\mathrm{d} x^{2}+\mathrm{d} y^{2}+\mathrm{d} z^{2}$, considering $\mathrm{d} x_{i}$ as variables and taking increments we get

$\delta(\mathrm{d} l)=\frac{\mathrm{d} x_{i}}{\mathrm{~d} l} \delta\left(\mathrm{d} x_{i}\right)$

so the second integral in Eq. (5) becomes:

$\int_{A}^{B} n_{\lambda} \delta(\mathrm{d} l)=\left.n_{\lambda} \frac{\mathrm{d} x_{i}}{\mathrm{~d} l} \delta x_{i}\right|_{A} ^{B}-\int_{A}^{B} \delta x_{i} \frac{\mathrm{d}}{\mathrm{d} l}\left(n_{\lambda} \frac{\mathrm{d} x_{i}}{\mathrm{~d} l}\right) \mathrm{d} l$.

Since the different considered trajectories start in the fixed points $A$ and $B, \delta x_{i}(A)=0$ and $\delta x_{i}(B)=0$, so Eq. (7) results as follows:

$\delta \Lambda=\int_{A}^{B}\left[\frac{\partial n_{\lambda}}{\partial x_{i}}-\frac{\mathrm{d}}{\mathrm{d} l}\left(n_{\lambda} \frac{\mathrm{d} x_{i}}{\mathrm{~d} l}\right)\right] \delta x_{i} \mathrm{~d} l=0$.

This equation must be true for any value of $\delta x_{i}$, which lets us come up with the equation to obtain the trajectory of a light ray in an inhomogeneous medium with a known index of refraction, which is

$\frac{\mathrm{d}}{\mathrm{d} l}\left(n_{\lambda} \frac{\mathrm{d} \vec{r}}{\mathrm{~d} l}\right)-\nabla n_{\lambda}=0 \leftrightarrow \frac{\mathrm{d}}{\mathrm{d} l}\left(n_{\lambda} \frac{\mathrm{d} x_{j}}{\mathrm{~d} l}\right)-\frac{\partial n_{\lambda}}{\partial x_{j}}=0$,

where $l$ is the length of the arc, $n$ is the index of refraction of the medium and with $(j=1,2,3)$ are the coordinates of the point. Since the index of refraction $n$ can be obtained for each point in space (see Section 3.2), the equation can be solved numerically. Naive resolution methods will yield huge computation times, so we need a robust, efficient numerical method. In this paper we apply the RK5(4)7M embedded Runge-Kutta method, from the DormandPrince family (see $[18,19])$. Appendix A provides a detailed description of the implementation, whereas Section 5 provides an analysis of error and computation times.

\subsection{The atmosphere}

To model the atmosphere we start with the 1976 US Standard Atmosphere [20]. It is an average of pressure and temperature for various altitudes, which make up the ideal, steady state profile. The values selected in 1976 come from slight modifications of the values adopted in 1962.

Fig. 2 shows the temperature and pressure values for the first $34 \mathrm{Km}$ of standard atmosphere from the values of the Standard Atmosphere (intermediate values are obtained by interpolation). As it can be seen, temperature decreases with height at an approximate rate of $0.6^{\circ} \mathrm{C}$ per $100 \mathrm{~m}$. Under these conditions, air is perfectly stable and no unusual effect takes place. ${ }^{1}$

Starting with the standard values, we seek to obtain a profile of the index of refraction for any arbitrary atmosphere and as a function of wavelength, in order to render the desired effects. We first need to obtain the density profile, for which we take the temperature and pressure data and apply the Perfect Gas Law:

$\rho(h)=\frac{P(h) M}{R T(h)}$,

where $\rho$ is the density we want to obtain, $T$ is the temperature, $P$ is the pressure, $M$ is the mean mass of the molecules of a mixed atmosphere $\left(28,96 \cdot 10^{-3} \mathrm{~kg} / \mathrm{mol}\right.$ typically) and $R$ is the gas constant, $8,314510 \mathrm{~J} / \mathrm{mol} \mathrm{K}$.

We now need an expression for the index of refraction in a medium as a function of wavelength. For low-density media (such as air), we can take Cauchy's dispersion formula [17]:

$n(\lambda)=a \cdot\left(1+\frac{b}{\lambda^{2}}\right)+1$,

\footnotetext{
${ }^{1}$ Actually, the sole presence of the atmosphere itself causes effects such as the astronomic refraction, which needs to be corrected for to accurately record the position of stars [21]; additionally, an ideal stationary state is never achieved, which reflects in the twinkling of distant lights at night.
} 

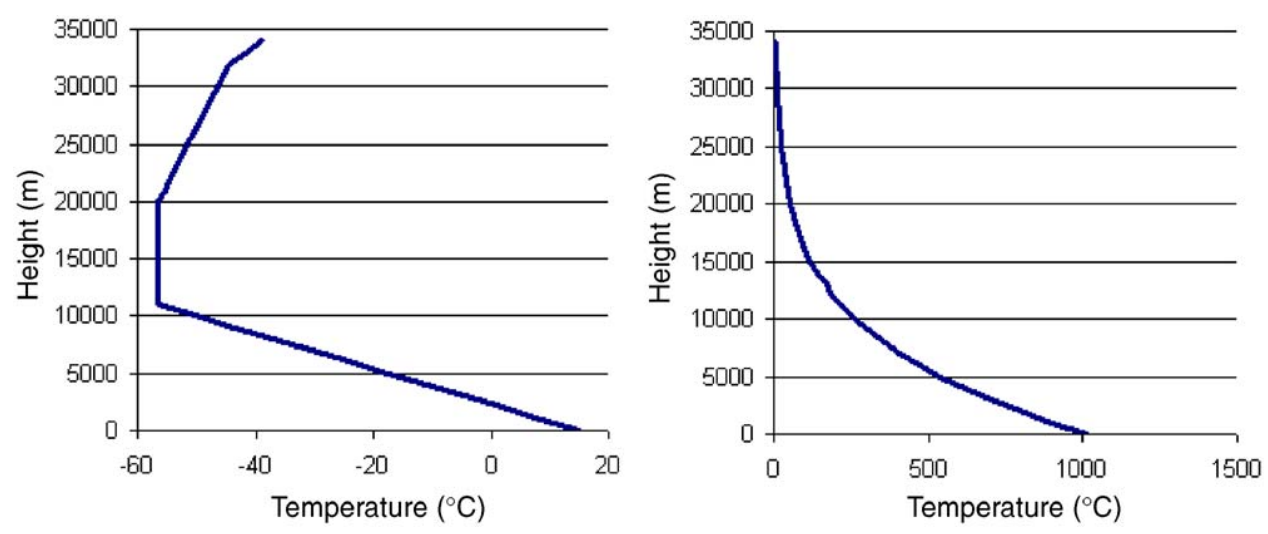

Fig. 2. Left: temperature profile of the USA 1976 Standard Atmosphere. Right: pressure profile of the USA 1976 Standard Atmosphere.

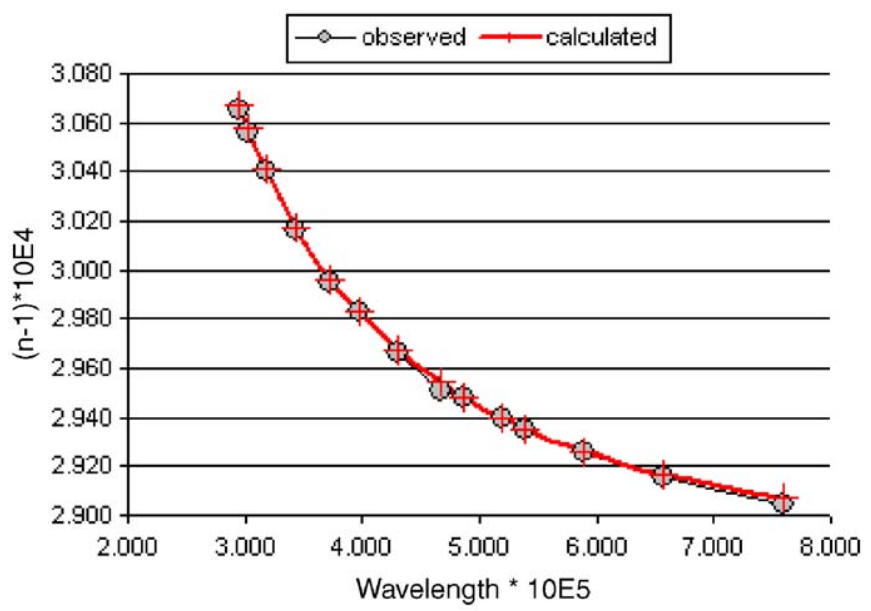

Fig. 3. Observed values of $n(\lambda)$ for air, and the values predicted by Eq. (11) (observed data after [17]).

where $a$ and $b$ are constants which depend on the medium. In case of air $a=28.79 \times 10^{-5}$ and $b=5.67 \times 10^{-5}$. This formula yields good approximations between the observed and the calculated $n(\lambda)$ for air, with errors of the order of $10^{-3}$. Fig. 3 shows a comparison between actual measured data and the values predicted by the formula.

At this point we have on the one hand density as a function of height (Eq. (10)) and on the other hand the index of refraction $n$ as a function of wavelength (Eq. (11)). To obtain $n$ for any height and any wavelength we should combine both equations, which can be done by applying the Gladstone-Dale formula [22]. The formula describes how refractivity (refraction index minus 1) is proportional to the density according to the following equation:

$n(h, \lambda)=\rho(h) \cdot(n(\lambda)-1)+1$.

Eq. (12) is the final expression which allows us to describe an exact profile for the index of refraction in any atmosphere, as a function of wavelength and starting with pressure and temperature data. The effect of humidity can easily be incorporated to our model, in which case the index of refraction can be obtained by using the formula

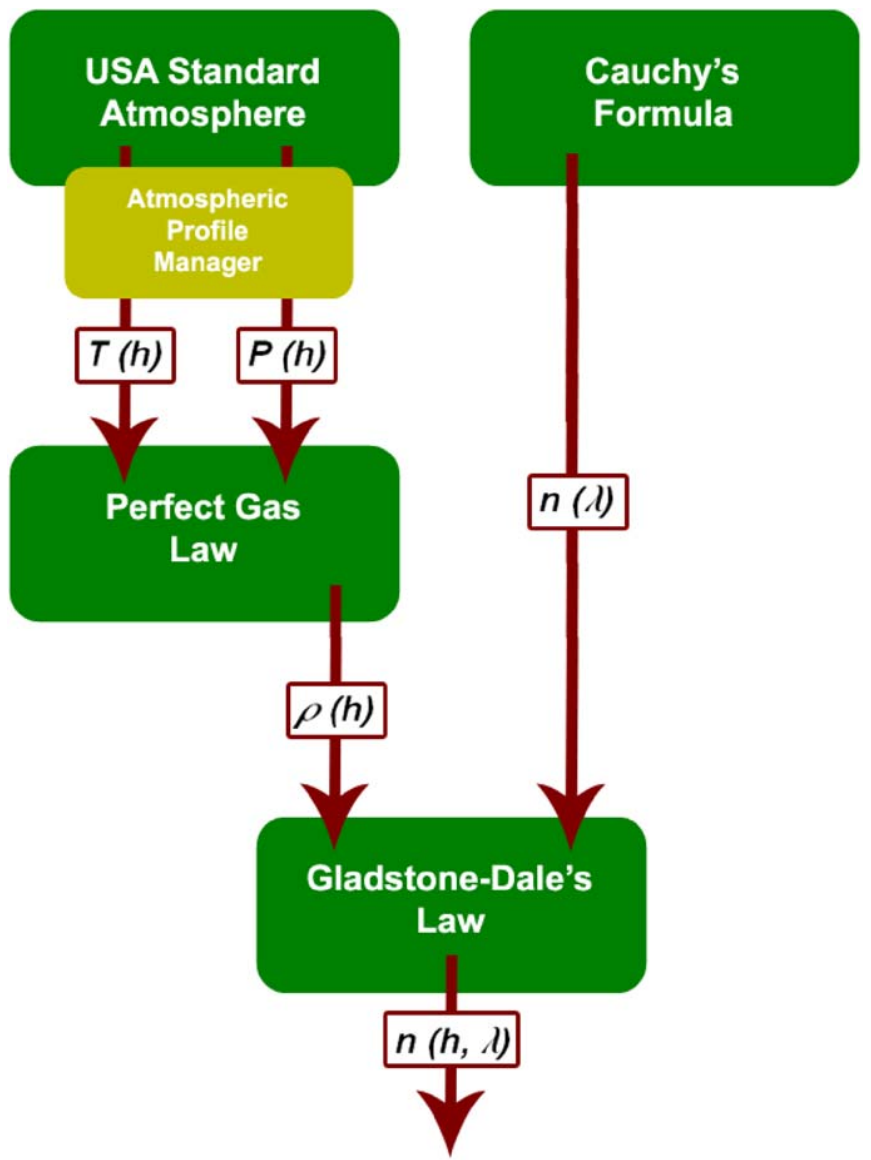

Fig. 4. Diagram of the process to obtain the index of refraction of the atmosphere.

proposed by Ciddor in [23], and using the modified US 1976 Standard Atmosphere, as proposed by van der Werf [24]. A full schema of the derivation of the atmosphere model can be seen in Fig. 4 (including the APM, described in the following subsection).

\subsection{De-standardizing the atmosphere}

A description of a standard atmosphere is a necessary starting point in that it ensures that the simulation is based 
on actual data. The next step in the simulation process is to de-standardize that atmosphere, recreating the conditions necessary for the effects to occur. To do that we have developed the APM, which enables to set different temperature and pressure profiles at different heights. From there, a new profile for the index of refraction is obtained as explained in the previous subsection (see Fig. 4), and the resulting curved light paths will yield the desired images. Since the non-standard profiles for atmospheric phenomena generation are always close to ground level (with a maximum altitude of $500 \mathrm{~m}$ ), we consider the rest of the atmosphere to remain standard, thus speeding up calculations.

Three methods have been developed for de-standardization, all of them based on previously published data: inversion layers, hot spots and noise grids. Each of these methods is focused towards the intuitive and efficient characterization of the atmosphere for a given set of phenomena.

\subsubsection{Inversion layers}

Most mirages and similar atmospheric phenomena are due to inversion layers. In the normal situation, the temperature decreases with height during the first thousand meters but under the right conditions inverted temperature gradients are formed. These inversion layers are characterized by the height at which they occur and their inverse temperature gradient. To insert them into the standard profile, thus altering it, we need a method suitable for ray tracing which also offers enough flexibility to tweak the parameters describing the layers. We use an analytical function known as the Fermi distribution, as proposed by van der Werf et al. in [25] (see Eq. (13)):

$T(h, x)=T_{A T M}(h)-\Delta T(x)+\frac{\Delta T(x)}{1+\mathrm{e}^{\left(h-h_{\text {ciso }}(x)\right) / a(x)}}$,

where $x$ is the distance in the direction parallel to the Earth's surface, $h_{\text {ciso }}(x)$ is the height of the inversion layer about which the added temperature profile is centered, $\Delta T(x)$ is the temperature jump across the inversion and the diffuseness parameter $a(x)$ determines the width of the jump (see Fig. 5). This distribution yields very accurate results, as can be seen in another work by van der Werf in which the author uses the same techniques [24] and compares the predicted atmospheric profiles with wellestablished standards such as the Star Almanac Atmosphere [26], the Nautical Almanac Atmosphere [27] and the Pulkovo tables [28]. Fig. 6, top right, shows a proof-ofconcept rendering of the distortion caused by an inversion layer.

The Fermi distribution is also suitable for animation, by allowing several parameters to be keyframed:

- $h_{\text {ciso }}(x)$ can be modified by a certain time-based function $f(t)$ by the additive expression $h_{\text {ciso }}(x, t)=$ $h_{\text {ciso }}(x)+f(t)$. Animating this parameter shifts the mirage vertically.

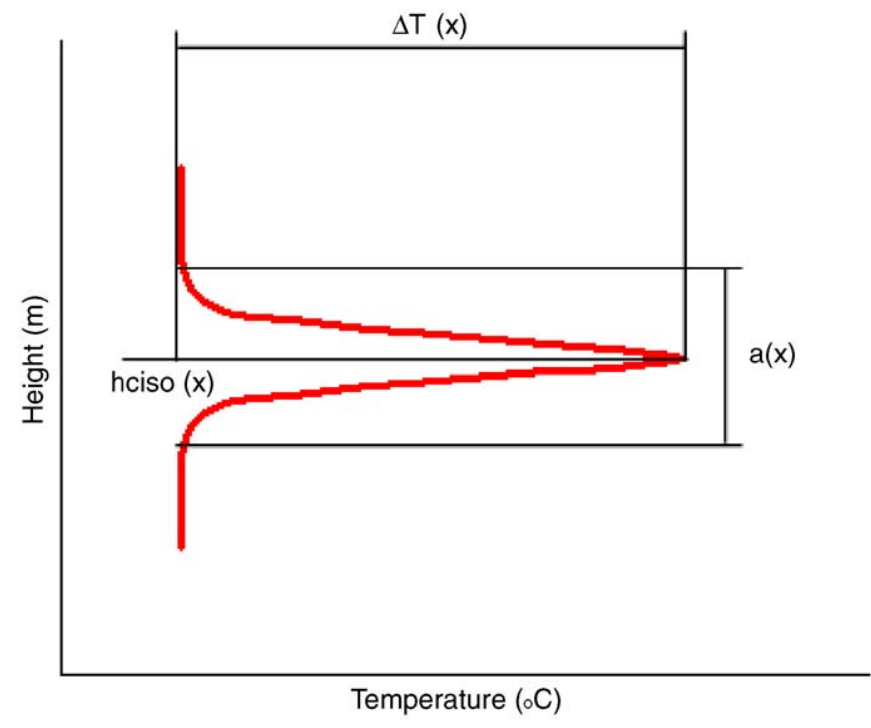

Fig. 5. Graph of the application of an inversion layer.

- $a(x)$ can be scaled by another time-based function $f^{\prime}(t)$ by using the expression $a(x, t)=f^{\prime}(t) a(x)$. This parameter can soften or sharpen the mirage.

- $\Delta T(x)$ can be scaled also by a factor $f(t)$ by the expression $\Delta T(x, t)=f(t) \Delta T(x)$. This is maybe the most interesting parameter to animate, as it can show how the simulated effects form over time.

\subsubsection{Hot spots}

Some effects can be bound to some local region of space. In the case of a road mirage this region would be the asphalt of the road itself (much hotter than its surroundings). To allow for that type of situations, we define a hot spot as an increase in temperature at a certain position in space, which affects its nearby region. To obtain the new atmospheric profile as influenced by the hot spot we first need to obtain the new temperature distribution created by it; we use the formula proposed by Stam [29] (Eq. (14)), which is based on experimental data found in the optical sciences literature [30]:

$T=T_{0}+\left(T_{s}-T_{0}\right) \exp \left(-d / d_{0}\right)$,

where $T_{S}$ is the known temperature of the hot spot, $d$ is the distance to the hot spot, $T_{0}=273 \mathrm{~K}$ and $d_{0}$ is the dropoff length (for the example mentioned above, the dropoff length for the heat field generated by a hot asphalt road is approximately one centimeter, as stated in the book by Minnaert [31]).

The concept of hot spot can be extended to other geometric elements, like lines, planes and even curves, surfaces or volumes. The only variation on Eq. (14) would be the substitution of $d$ by a more complex expression of the distance to the chosen element; however, experience says that hot spots are usually enough for modeling all localized effects. Therefore, our current version of the APM only implements hot spots, although it can be easily 

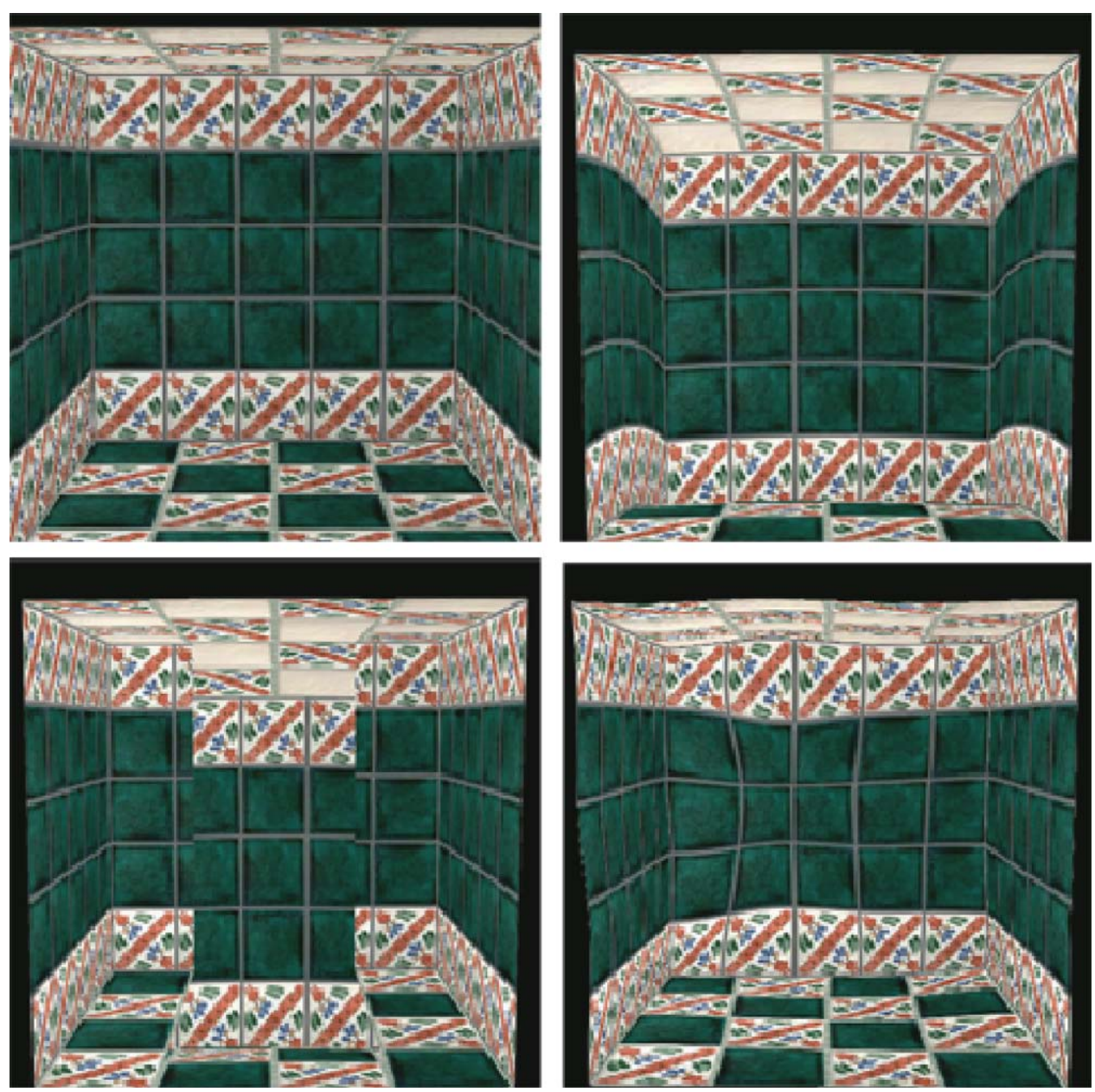

Fig. 6. Concept pictures of the distortions caused by the proposed de-standardization methods. Top-Left: no distortions (homogeneous medium). Topright: inversion layers. Bottom-left: hot spots. Bottom-right: noise grids.

extended in the future to other geometric elements. The application of the localized effect can be seen in Fig. 6, bottom left. This definition of a hot spot is also suitable for animations.

\subsubsection{Noise grids}

Noise grids have also been added to the APM. They enable the simulation of effects caused by unstable heat waves, coming for instance from a hot car engine or an activated jet engine (see Fig. 6, bottom right). Noise grids are $3 \mathrm{D}$ regions of space where temperature varies randomly between a defined maximum and minimum. A random $\Delta T$ value is set at each vertex of the grid, and the values inside the grid are interpolated by Hermite interpolation. The effect can be animated by adding time as a fourth dimension to the grid. The result is similar to the rippling effect caused by extremely hot surfaces.

Effects generated by noise grids could also be generated by a set of hot spots, but they are a very useful tool in the APM for fast modeling of atmospheric profiles for certain effects. A combination of both is also possible: for instance, to simulate distortions caused by fire, a noise grid can provide the general turbulence whereas hot spots can keep the effect localized to those parts of the scene close to the fire.

\section{Rendered scenes}

We are now going to use our curved ray tracing algorithm to obtain rendered images of some atmospheric effects. The most important step in our simulations is to obtain a correct temperature profile, and to derive from it an accurate index of refraction profile for the whole atmosphere, which will curve the rays as they traverse the medium. For our simulations we will only model an atmosphere of $40 \mathrm{~km}$ : from there up the refraction effects can be neglected, greatly reducing computation times without visible effects in the rendered image.

We have designed scenes with very simple geometry, but with very accurate atmospheric profiles and using realworld distances and dimensions. The Earth is always modeled as a sphere of 6371 units of radius (obviously one unit in the $3 \mathrm{D}$ scene equals $1 \mathrm{~km}$ in the real world). A slightly bigger, concentric sphere forms a $40-\mathrm{km}$ atmosphere layer. The rest of the setup of the scenes is characteristic of each different simulated effect. 
The simplicity of the whole setup is irrelevant, though, since it is the curvature of the ray traversing the medium the only factor that matters. This correct curvature is guaranteed by (a) a precise atmosphere profile, (b) using real geometric dimensions and (c) accurately solving the physically based equation that governs the paths of the light rays.

All the simulations are directly compared with real pictures. It is important to note that the whole setup has had to be deduced from just a picture and based on trial and error and educated guesses based on experience. Although thanks to the APM this trial and error is much faster and more efficient, it is virtually impossible to reproduce the exact atmospheric conditions of the real scene when the picture was taken, using only the information contained in the snapshot. Some differences between the real picture and the rendered image are therefore to be expected, since accurate data at the moment of the picture is not available anywhere.

\subsection{The Viking's end of the world}

We begin our exploration with the ancient arctic legend of the Viking's end of the world. They believed that there lay a land at the end of the world, a land they called Ultima Thule, where all ocean waters flowed back to the known lands. According to several sources, the word Thule comes from the Celts, and means "to raise oneself". Vikings also believed in massive sea walls that would raise over the horizon as one approached Ultima Thule [32].

Maybe the legend was born from a very scientific fact: the distortion of the horizon which occurs under very common polar atmospheric conditions. When the temperature of the lower atmosphere rises at a rate of $11.2^{\circ} \mathrm{C}$ per $100 \mathrm{~m}$, the light rays bend in an arc exactly equal to the curvature of the Earth, and the horizon will appear flat, like an infinite plane. If the inversion gradient becomes even stronger, the light rays exceed the curvature of the

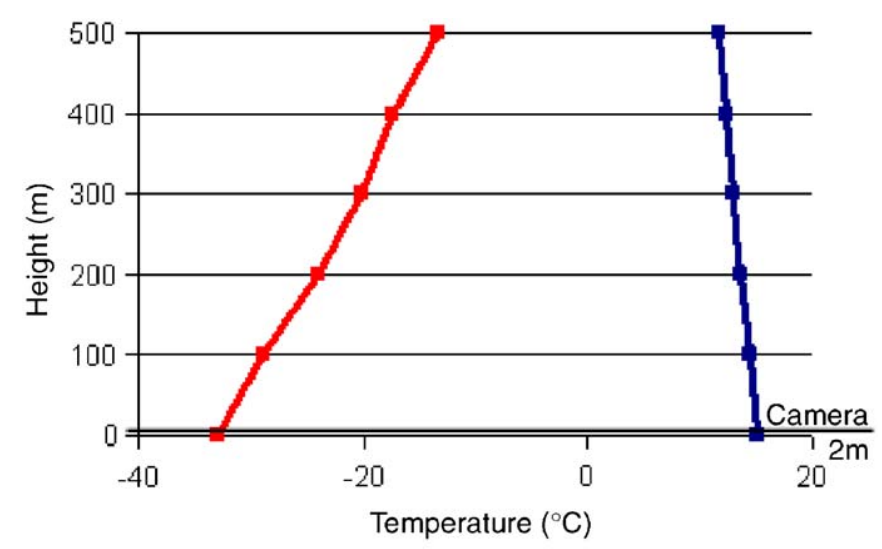

- Ultima Thule protile - USA. Standard Atmosphere profile

Fig. 7. Temperature profile of the Viking's end of the world effect compared to the USA Standard Atmosphere's temperature profile.
Earth, and the horizon will appear to rise vertically from the flat position.

To recreate this, we have covered the Earth sphere with a tiled texture of the Northern Bering Sea, and given the atmosphere sphere a grayish sky color. The index of refraction varies continuously with height, with a temperature profile as seen in Fig. 7. Fig. 8 (left) shows the scene rendered with a standard ray tracer: no curvature at all has been applied to the rays, and therefore the horizon appears undistorted. Fig. 8 (right), on the other hand, shows how the horizon gets in fact distorted when taken into account the real paths that light travels in a polar atmosphere. The horizon line clearly rises above its real location, while getting slightly curved upward.

\subsection{The inferior mirage}

The inferior mirage, also known as the "water on the road" effect, is probably the best known atmospheric effect owed to curved light paths. ${ }^{2}$ This effect occurs when there is a strong decrease of temperature with increasing height. As a consequence, the light rays approaching the ground are curved upwards, generating an inverted image of the object in the ground. The inferior mirages tend to shake and change rapidly since hot air is less dense than cold air, and it is therefore too unstable to remain static. Contrasting with this, superior mirages (see Section 4.3) are very stable since a denser cold mass at the bottom will remain mostly static.

Fig. 9, left, shows a picture of a real inferior mirage. Fig. 9, right, shows a simulation with our system of the same phenomenon (differences owed to the lack of accurate data when the picture was taken). The shuttle seems to be reflected as if the ground level was a mirror. The temperature profile used for the rendered image can be seen in Fig. 10, while Fig. 11 shows an animation of the inferior mirage as it is formed.

\subsection{The superior mirage}

In 874 the Norse Vikings settled in Iceland. A century later, a former chief named Erik the Red was banished from Iceland, and he and his crew set sail around 982 to find another place to live. They ended up in Greenland, which surprisingly is not visible from Iceland owed to the Earth's curvature. What made him head in that direction? ${ }^{3}$

Given the right conditions, it is possible for objects lying beyond the horizon to actually be seen [34], in a manifestation of the superior mirage. The object itself will remain hidden by the Earth's curvature, but its mirage will be seen above the horizon. Also known by its Icelandic word as the hillingar effect, the superior mirage occurs when there is an increase of temperature with height near

\footnotetext{
${ }^{2}$ The word mirage comes from the Latin mirari, "to be astonished".

${ }^{3}$ Other historical references regarding superior mirages can be found in [33].
} 

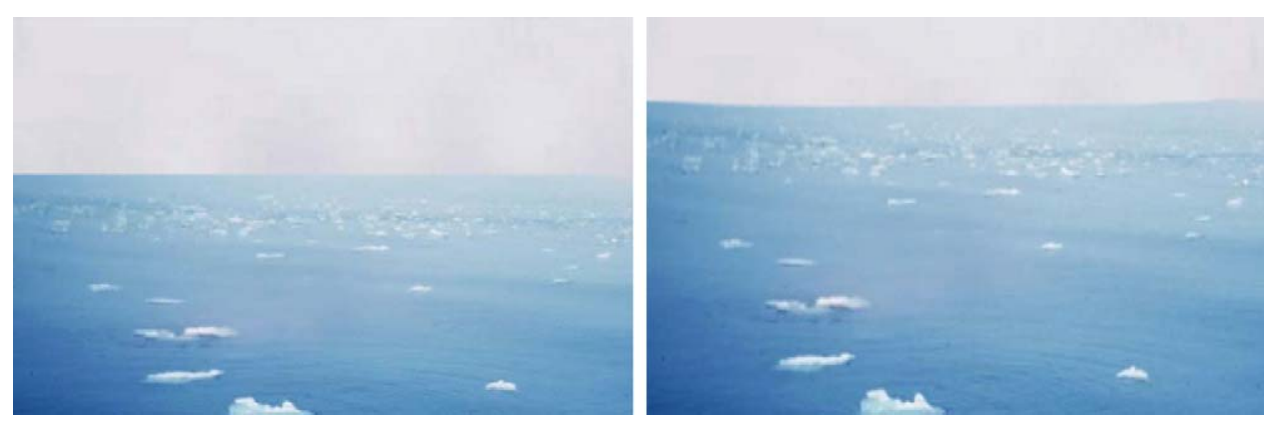

Fig. 8. Left: Northern Bering Sea, rendered without ray curvature. Right: rendered image of the distortion in the horizon caused by a polar atmosphere.
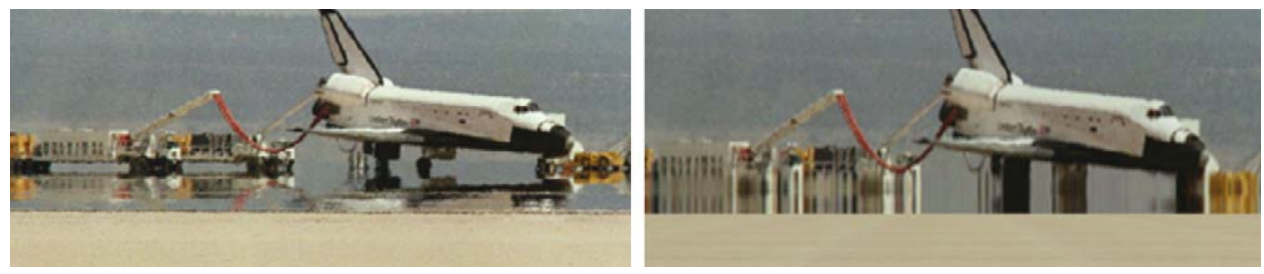

Fig. 9. Left: real picture of an inferior mirage. Right: rendered inferior mirage.

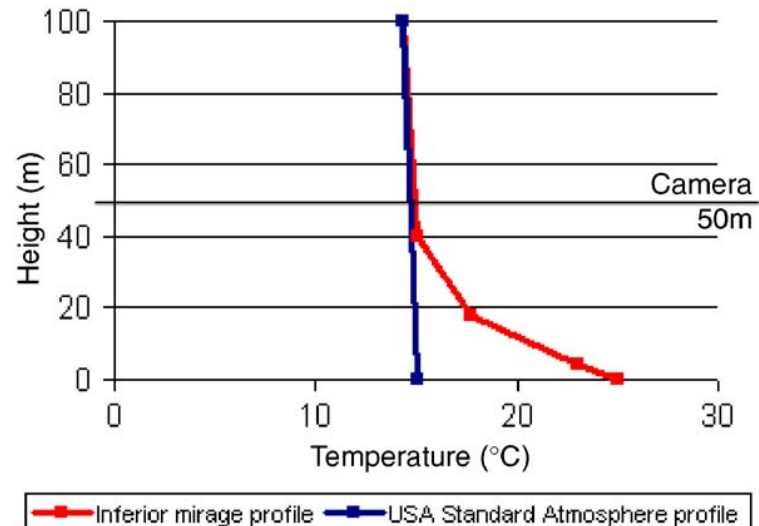

Fig. 10. Temperature profile of the inferior mirage rendered in Fig. 9 compared to the USA Standard Atmosphere's temperature profile.

the ground, meaning there is an inversion layer. As a consequence, the light rays approaching the ground are curved downward. They are also known as arctic mirages, since the images are widely seen in arctic latitudes.

Depending on the gradient of the inversion layers, the arctic mirage can offer an inverted or an upright view of the real object. Fig. 12, left, shows a real picture of a ship, with the simulated image on the right. ${ }^{4}$ Again, some discrepancies are to be expected owed to lack of reliable data. The simulation was done with the ship located at $2.6 \mathrm{~km}$ from the viewer, with the camera at $10 \mathrm{~m}$ above ground level. The temperature profile can be seen in Fig. 13. Fig. 14 shows the superior mirage as it forms over time.

\footnotetext{
${ }^{4}$ It is interesting to note how this could explain the countless ghost ship stories told centuries ago by highly superstitious mariners.
}

\subsection{The Fata Morgana}

Fata Morgana is the name of a fairy enchantress skilled in the art of changing shape, and literally means "the fairy Morgan" in Italian. One of the versions of the legend makes her King Arthur's sister and explains how she learned many of her skills from Merlin the Magician. She lived in a castle under the sea, and sometimes she would make her castle appear floating in the sky [35].

The mirage known as Fata Morgana occurs when there are several alternating cold and warm layers of air near the ground or the sea. These alternating layers cause a multiple concatenation of inferior and superior mirages, resulting in a complicated superposition of images of the same object (Fig. 15, left). The number of inversion layers usually varies between two and five, with the images alternating between upright and inverted. In addition to this, slight up-anddown movements of the air layers make the images change shape and apparent size. Even though it is mostly seen in polar latitudes, its Italian roots are owed to the fact that it was first described in the Strait of Messina, between continental Italy and Sicily. Fig. 15 (right) shows our raytraced image, using the temperature profile seen in Fig. 16. Fig. 17 shows our animation of the Fata Morgana effect.

\subsection{The Novaya-Zemlya mirage}

In the winter of 1596, Dutch explorer Willem Barents and his crew were sailing off the north coast of the Russian arctic island of Novaya-Zemlya. They were forced by thick arctic sea ice to spend the winter there, submerged in the long arctic night. One day in early March they were surprised to see a distorted sun appear for a short time 

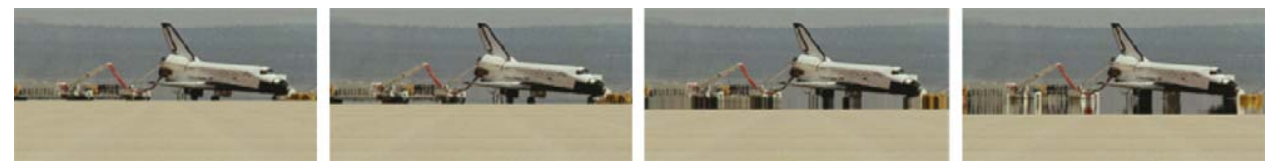

Fig. 11. Frames of an animation of the inferior mirage.
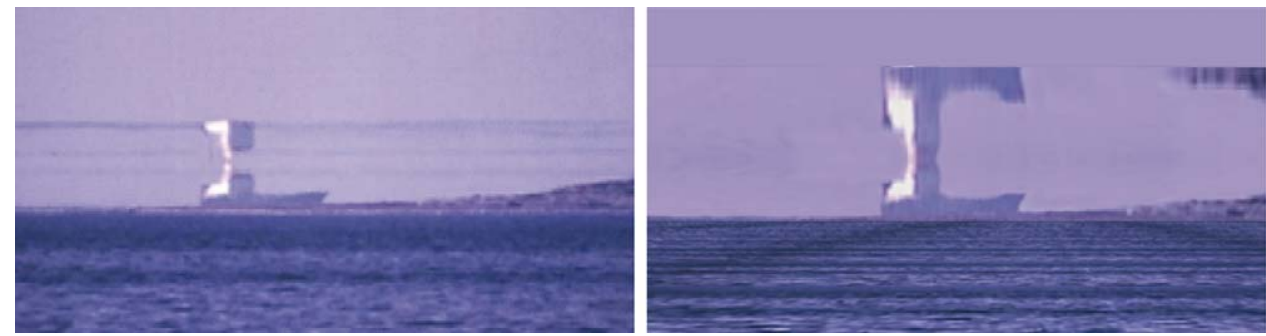

Fig. 12. Left: real picture of a superior mirage. Right: rendered superior mirage.

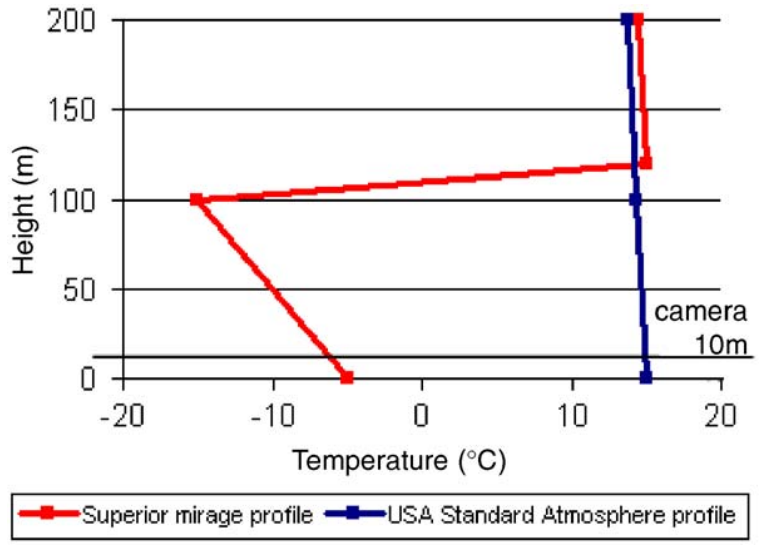

Fig. 13. Temperature profile of the superior mirage rendered in Fig. 12 compared to the USA Standard Atmosphere's temperature profile.

above the horizon, almost two weeks ahead of time, bringing an early twilight to the island [36].

What they saw was the Novaya-Zemlya mirage [25], which describes a case when celestial objects, such as the sun, can be seen even when they are situated below the horizon. The conditions for the effect differ from those of the superior mirage. Steep arctic temperature inversions trap the sunrays, making them travel within an inversion layer for hundreds of kilometers (a phenomenon known as ducting). If the layer has the right temperature gradient, the light bends following the curvature of the Earth over that long distance, effectively showing up above the horizon, even though the sun might really be up to $5^{\circ}$ below it.

With long-term scientific polar settlements established over the past few decades, this phenomenon has been widely photographed. Once the sun has burst above the horizon, its light still remains trapped within the inversion layer, distorting it into an unusual rectangular shape. Fig. 18 shows a real Novaya-Zemlya effect, alongside our ray-traced image. Fig. 20 shows our animation of the Novaya-Zemlya effect.
The atmospheric profile can be seen in Fig. 19. The sun has been modeled as a circle with a $1392000-\mathrm{km}$ diameter, placed 150 million kilometers away from the camera. In order to get a better view of the effect, the camera has been placed inside the inversion layer, meaning at $2 \mathrm{~m}$ from ground level.

\subsection{The green flash}

The green flash [37] is a real phenomenon (as opposed to an optical illusion as it is said in some texts), which occurs under very specific conditions both during sunrise and sunset. A small part of the sun will change its color, from red-orange to blue-green. lasting for about $2 \mathrm{~s}^{5}$

The cause of this effect is the dispersion of light as it traverses the atmosphere: the beam of white light gets split into all the different wavelengths. Since the index of refraction is a function of wavelength, the net result is that the paths of light are curved depending on the wavelength (Fig. 21). This dependance is so weak that most of the time remains unseen, hidden by Rayleigh or Mie scattering.

The explanation of the green flash effect is pretty straightforward once dispersion is taken into consideration: the closer the sun is to the horizon, the longer the light paths from the sun to the observer. Since the atmosphere is denser at low heights (its index of refraction is higher), light rays get bent downwards, and because of its wavelength dependency, blue and green rays are bent more than orange-red ones. The result is a green-blue halo on top of the solar disc, and a red rim under it (see Fig. 22 for a visual explanation). Aerosols in the atmosphere and Rayleigh's dispersion [5] eliminate low wavelengths (blue) so what remains is the green that gives the name to the

\footnotetext{
${ }^{5}$ Verne [38] explained the green flash as a green which no artist could ever obtain on his palette, a green of which neither the varied tints of vegetation nor the shades of the most limpid sea could ever produce the like! If there is a green in Paradise, it cannot be but of this shade, which most surely is the true green of Hope.
} 

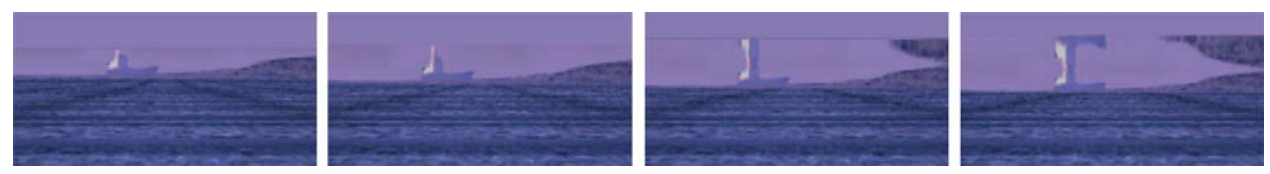

Fig. 14. Frames of an animation of the superior mirage.
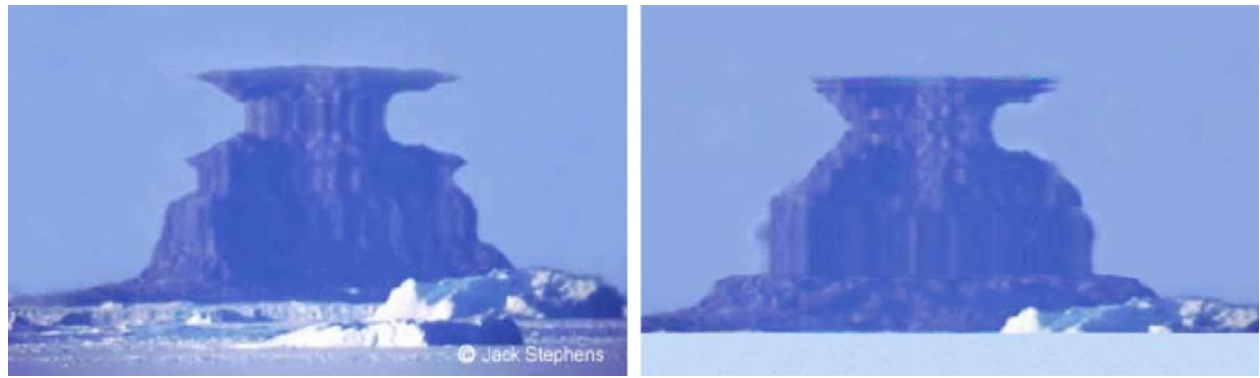

Fig. 15. Left: real picture of a Fata Morgana. Right: rendered Fata Morgana.

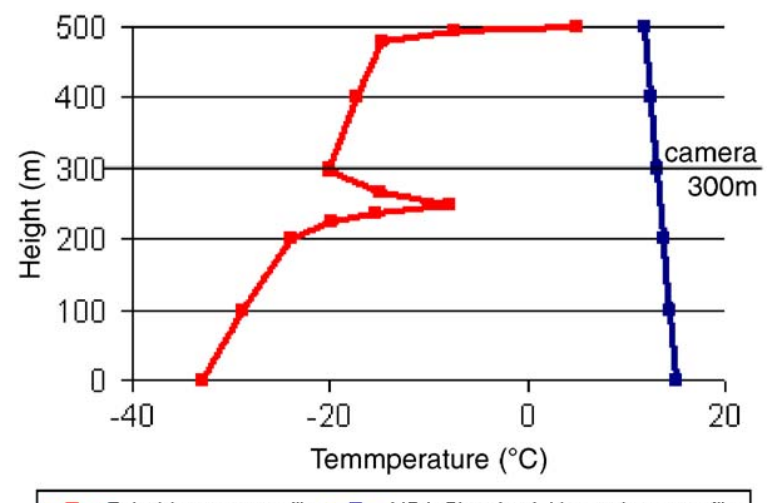

Fata Morgana profile - -USA Standard Atmosphere profile

Fig. 16. Temperature profile of the Fata Morgana rendered in Fig. 15 compared to the USA Standard Atmosphere's temperature profile.

green flash. A picture of the phenomenon can be seen in Fig. 23, left. The red rim of the green flash is usually invisible under the horizon, although it can be seen sometimes as in Fig. 23, right.

It is also true that under even more special atmospheric circumstances, others colors can be seen, as in Fig. 24. These colored flashes are due to the same atmospheric phenomena than the green flash itself. They are due also to Rayleigh's dispersion. Sometimes this dispersion is not strong enough and still some low wavelengths remain, so the flash can be blue or cyan. On the other hand, given the adequate circumstances, even medium wavelengths in the greenish range can be dispersed, the result being a yellow flash.

The green rim in the solar disc is too small for the naked eye to see, but its effect is usually magnified by some kind of mirage. Fig. 25 shows some frames of our simulation of the green flash, magnified by an inferior mirage. The atmospheric profile that has generated the green flash is shown in Fig. 26. Also Rayleigh's scattering and bleaching (see next section) have been considered.

\subsubsection{Bleaching}

The green flash is specially interesting since the effect is not only caused by the dispersion of the atmosphere (as presented before), but it is also magnified by our visual system. The retina of the eye is a compound of photoreceptors called rods and cones. Rods are very sensitive under low lighting conditions whereas cones are active in photopic luminance levels. According to the part of the visible spectrum to which they are most sensitive, the cones can be referred to as long-, middle- and shortwavelength-sensitive (L, M and S). When the light bleaches the pigment in a photoreceptor its density decreases. The loss of concentration in the photopigment makes the absorption of light diminish and the range of spectral sensitivity becomes narrower around the wavelength peaks. In cones, the effects of bleaching on pigment concentration can have large effects on spectral sensitivity.

When waiting for the green flash to occur, the retina has been exposed to very bright red light for relatively a long time. That bleaches some of the red-sensitive photopigments in the L-cones. This degeneration has a great effect on our spectral sensitivity, and the now less sensitive cones will perceive red as yellow, and yellow as green. Consequently, the green rim of the green flash will become even greener, as it can be seen in our tests simulations in Fig. 27. For a complete description of the model of bleaching used, the reader should refer to [39].

\section{Validation of the method}

As it has been stated before, the functions and formulas used to derive our atmospheric profiles are based on measured data, or a comparison between predicted values and real data has been performed. We have borrowed 

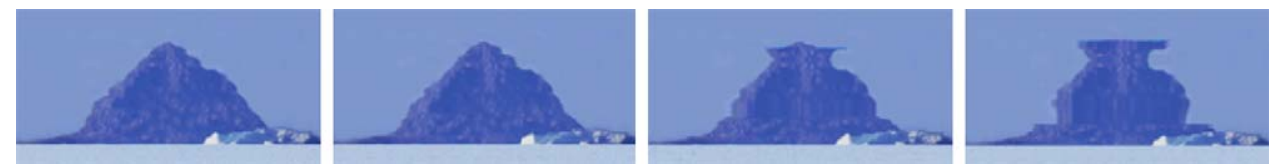

Fig. 17. Frames of an animation of the Fata Morgana.
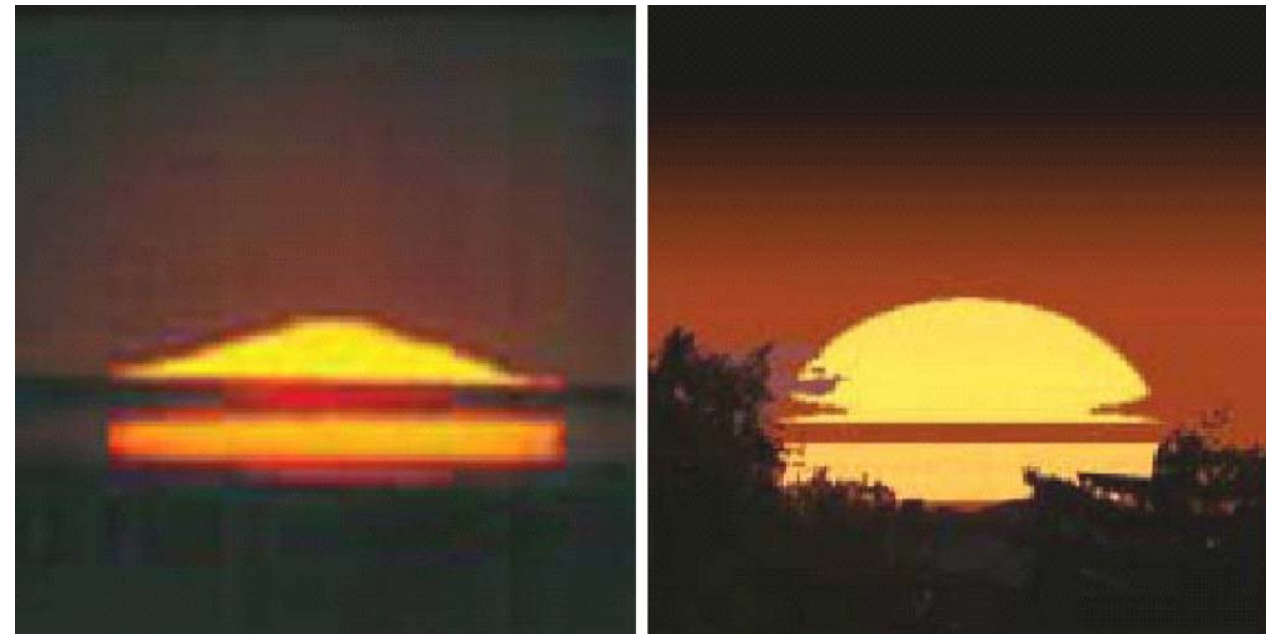

Fig. 18. Left: real picture of a Novaya-Zemlya effect. Right: rendered Novaya-Zemlya.

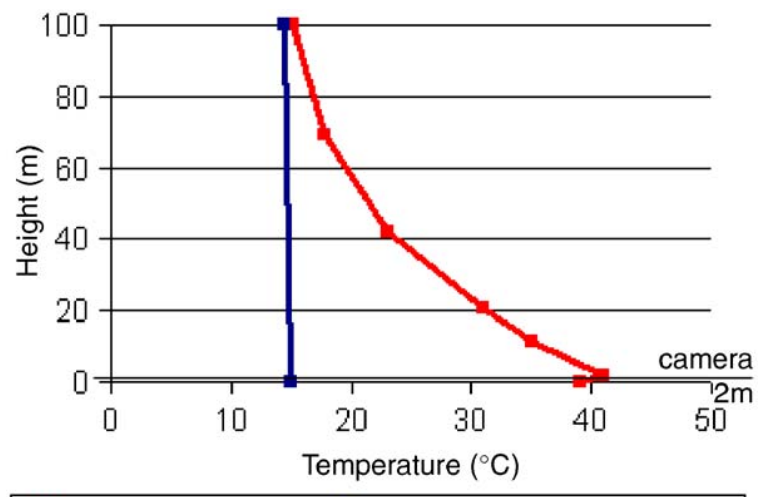

- Novaya-Zemlya protile $\rightarrow$ USA Standard Atmosphere protile

Fig. 19. Temperature profile of the Novaya-Zemlya effect rendered in Fig. 18 compared to the USA Standard Atmosphere's temperature profile.

extensively from scientific literature in other areas of research such as optics or meteorology, in order to ensure the predictability of the model.

To validate our resolution method, we have tested the implementation using a similar scheme than the proposed in [14], consisting on an empty scene where the index of refraction varies according to the equation $n=1+k y$, with $y$ representing height, and $k$ varying from -0.1 to 0.1 . This distribution of $n$ can be solved analytically, so we can measure the numerical error against the exact solution. The analytical and numerical solutions are compared shooting a ray into the scene and following it until it travels 100 distance units. We also analyze the results varying the error tolerance of the method. Fig. 28 shows the error of the
Dormand-Prince RK5(4)7M method as the tolerance is reduced, along with the time it takes to compute the solution. As it can be seen, error tolerances in the range of $10^{-8}$ to $10^{-12}$ yield good results without much of a time penalty. Error tolerances beyond $10^{-14}$ start increasing rendering times considerably. The validation tests were run on a P4 at $2.8 \mathrm{Ghz}$ and $1 \mathrm{~Gb}$ RAM.

In terms of convergence, the chosen Dormand-Prince method is orders of magnitude faster than simpler numerical resolution methods like Euler.

\section{Conclusions}

This paper presents a method for the simulation of some atmospheric phenomena owed to curved light paths. It is based both on the laws of physics and on actual measured data. It overcomes the limitations of previous works by using a physically based characterization of the index of refraction of the atmosphere as a function of wavelength, implementing an APM to characterize atmospheres based on measured data. Using real-world dimensions for the rendered 3D scenes, we obtain the curved paths of the rays by efficiently solving the differential equation that describes their trajectory, based on Fermat's Principle. It also includes a model of visual perception based on the bleaching of photoreceptor cells in the human eye, applied to the simulation of the green flash.

The resolution method has been validated numerically, with very low errors at virtually no cost. All the formulas used to create atmospheric profiles have been justified, borrowing data from meteorological studies previously 

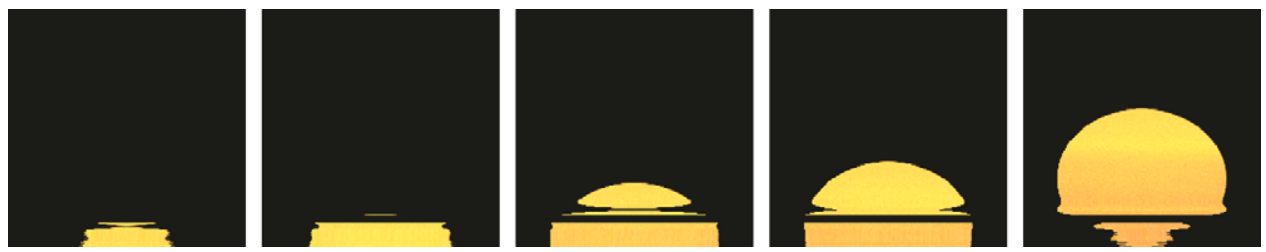

Fig. 20. Frames of an animation of the Novaya-Zemlya.
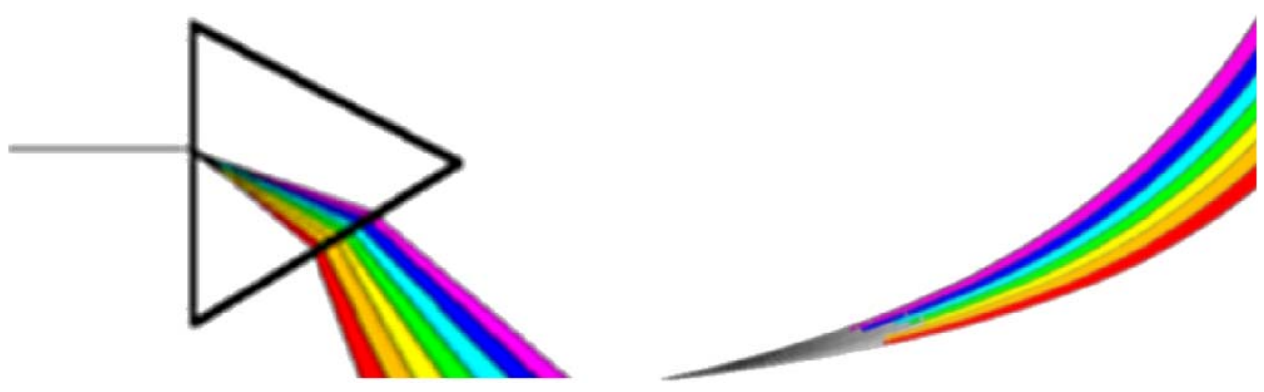

Fig. 21. Dispersion of light while traversing an inhomogeneous medium.
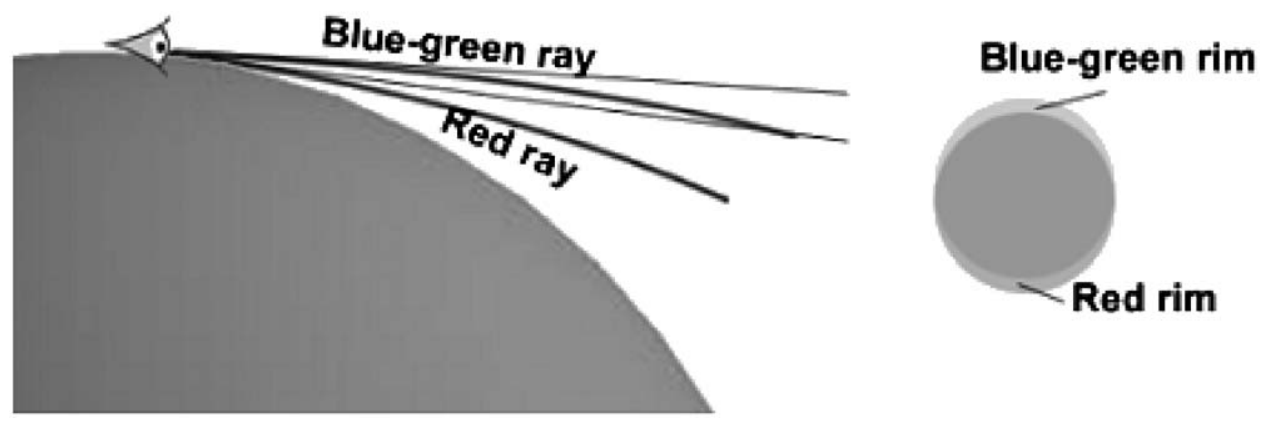

Fig. 22. Explanation of how atmospheric dispersion generates a green flash.
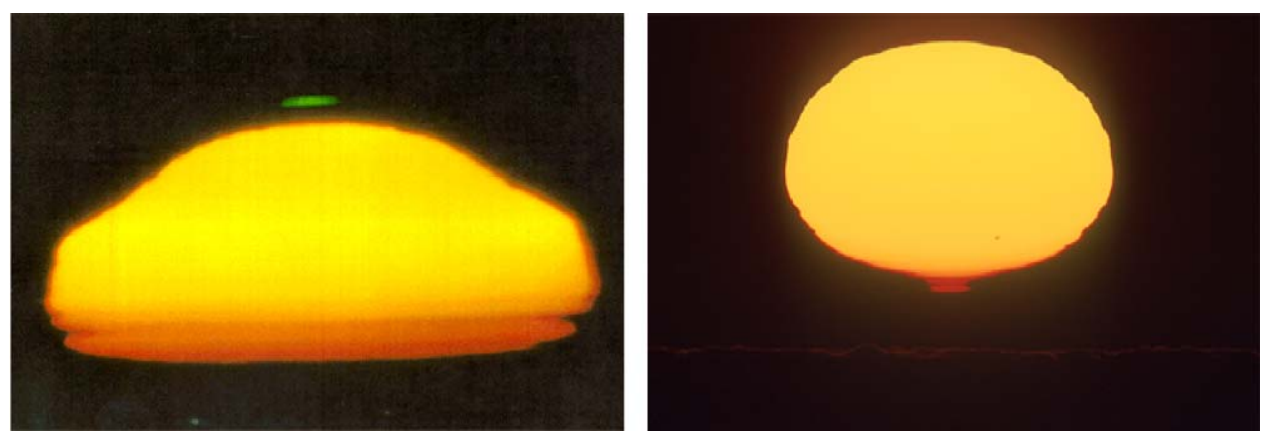

Fig. 23. Left: the green flash. Right: the red flash.

published in other areas of research. This makes this paper a true multidisciplinary effort, which we believe is essential in order to mimic nature in computer graphics.

We have simulated several of the most spectacular effects that are owed to light traveling through the atmosphere: the distortion of the horizon, the inferior and superior mirages, the Fata Morgana, the Novaya-Zemlya and the green flash, and propose others such as the rippling effect caused by heat waves or the distortions caused by fire. For rendering, we can assume the atmospheric profile to be symmetrical with respect to the $Y Z$-plane ( $X$ and $Y$ being the horizontal and vertical in the image plane, $Z$ being depth); this is a safe assumption since none of the effects simulated in the paper depend on asymmetries along the $X$-axis, except for the distortions caused by fire. Therefore, only half of the curved paths need to be numerically calculated; the other half can be obtained by mirroring those curved paths, reducing rendering times in almost half. 

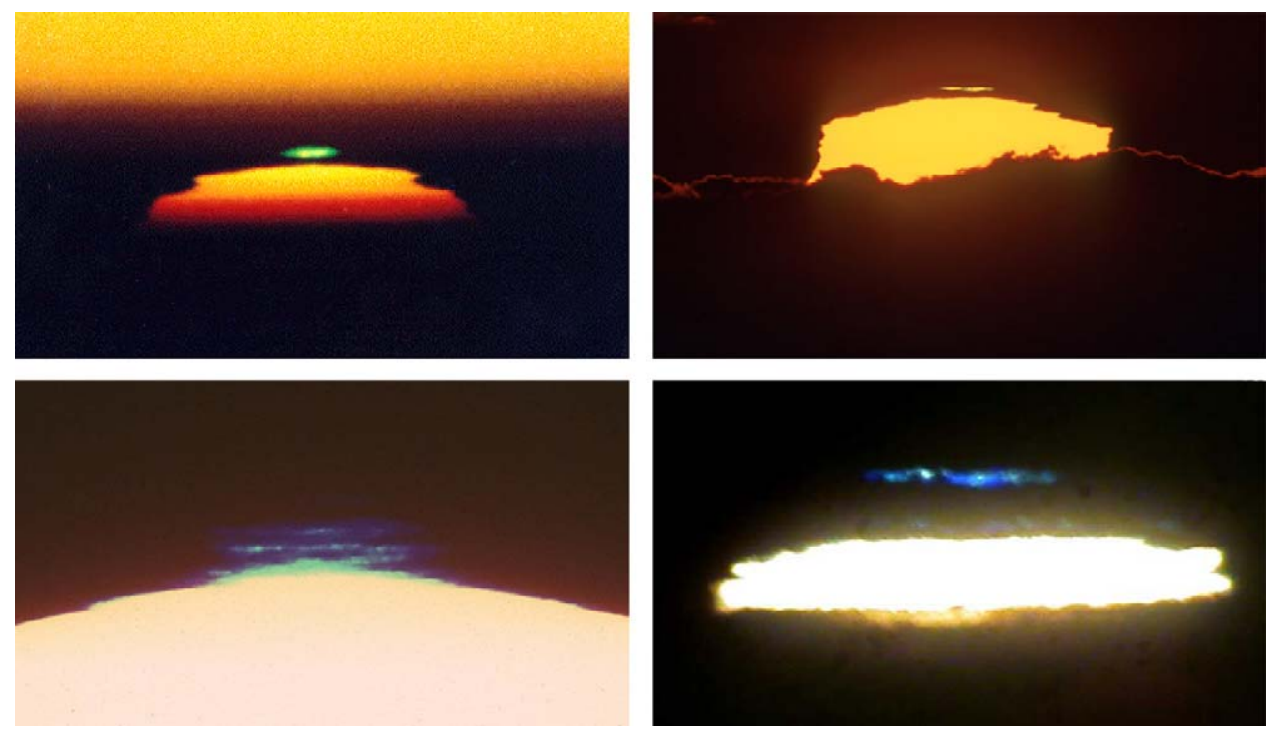

Fig. 24. Different colors of the green flash: green, yellow, cyan and blue.
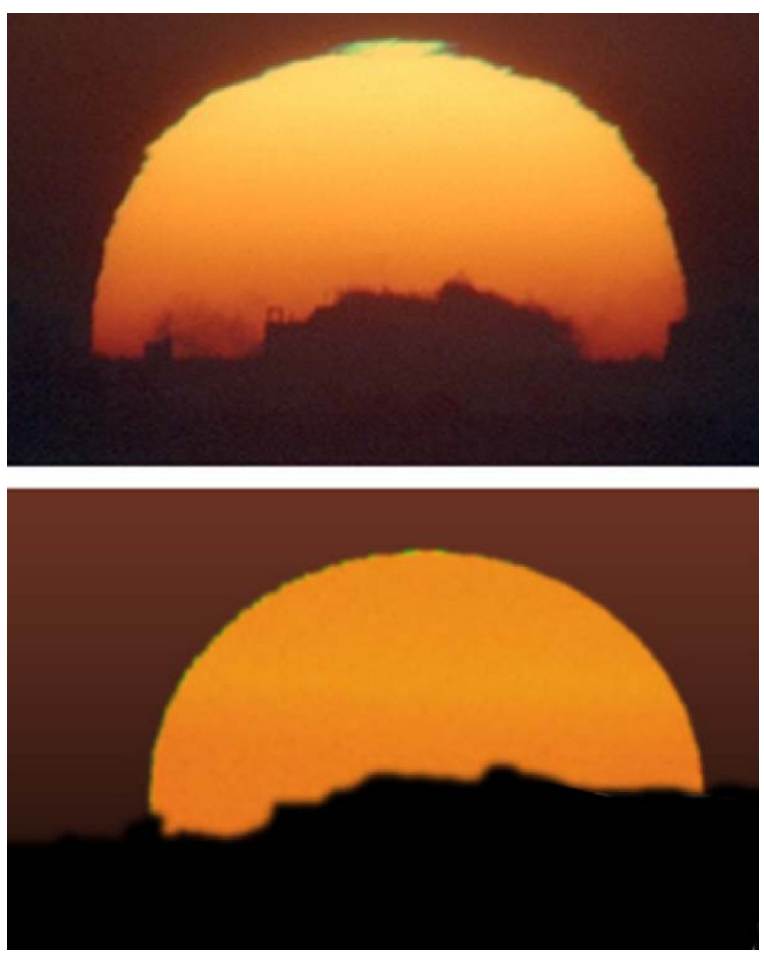

Fig. 25. Our simulation of the green flash.

The distortion of the horizon, the Novaya-Zemlya effect and the heat waves effect were rendered at $400 \times 400$ pixel resolution, one ray per pixel, on a Pentium IV at $2.8 \mathrm{Ghz}$. All of them took around $2.5 \mathrm{~min}$ per frame. The green flash was rendered at the same resolution but sampling eight wavelengths between 320 and $720 \mathrm{~nm}$ and adding the bleaching effect, and it took an average of 5 min per frame. On the other hand, the mirages and the Fata Morgana were rendered at $800 \times 400$ pixel resolution, one ray per pixel, on a Pentium IV at $2.8 \mathrm{Ghz}$. They took between 4

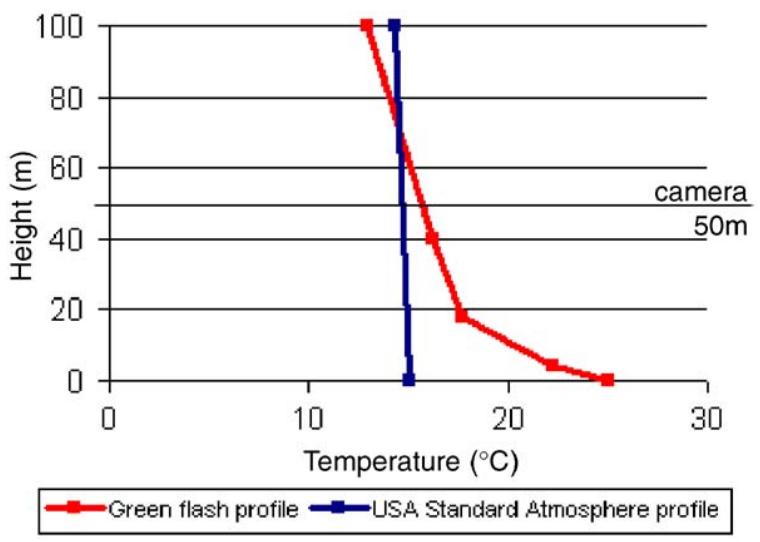

Fig. 26. Temperature profile of the green flash effect rendered in Fig. 25 compared to the USA Standard Atmosphere's temperature profile.

and $5 \mathrm{~min}$ per frame. The error tolerance for the simulations was set to $1 \mathrm{~cm}$.

Computation times can be drastically reduced in the case of orthogonal rendering: if no perspective is calculated (and $Y Z$-symmetry is still assumed), the curved paths need to be obtained for only one column of pixels, replicating the deformations for the rest of the columns. This can be useful in some image-based rendering contexts [40], and the rendering times for a $800 \times 400$ pixel image will therefore be reduced by a factor of 800 , thus achieving interactive rates.

\section{Future work}

Still lots of work lay ahead. The number of light effects owed to inhomogeneous media does not end with the ones presented here. Adding polarization effects to our system, we could also aim at obtaining halos, sun dogs or glories [41]. 

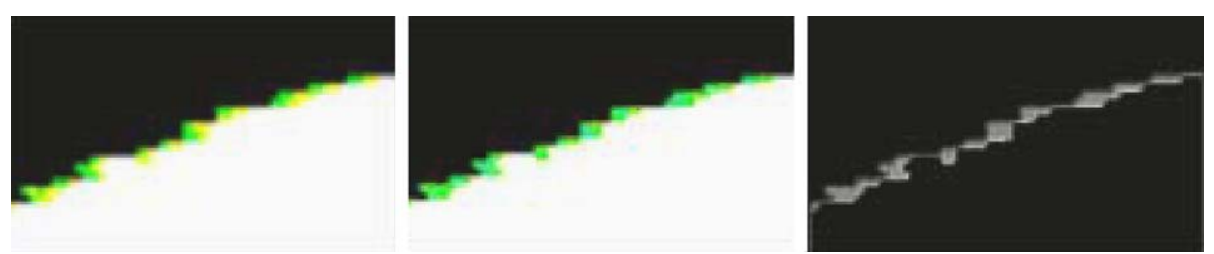

Fig. 27. Left: a simple test simulation of the green rim of a green flash. Middle: the same simulation after the application of the bleaching algorithm. Right: green pixels enhanced by bleaching.
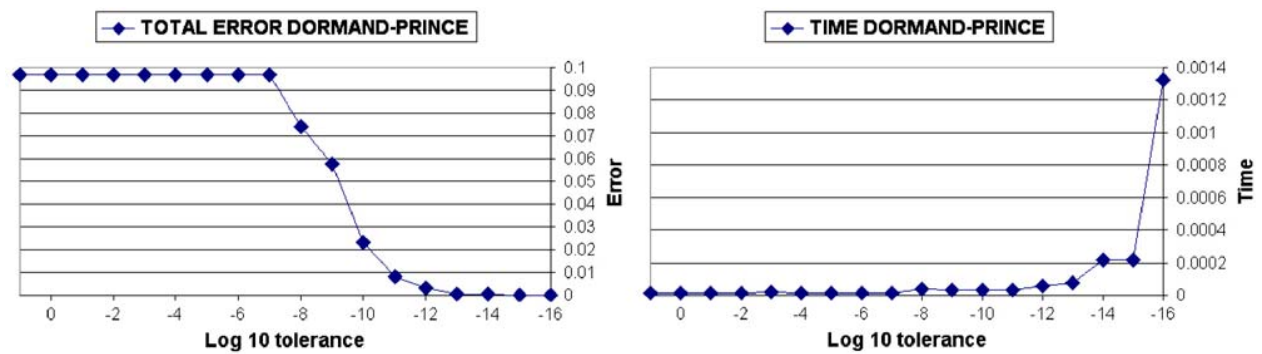

Fig. 28. Error and rendering time (s) as functions of the error tolerance in the Dormand-Prince RK5(4)7M method for a test scene.

For animations of the effects, further research on the dependency of the index of refraction with time needs to be carried out.

Finally, we are currently working on a parallel version of this work, in order to speed up rendering times.

\section{Appendix A. Implementation of the Dormand-Prince method}

We chose to use a Runge-Kutta method to solve Eq. (9). Expressing such equation in components we get:

$\frac{\mathrm{d}}{\mathrm{d} l}\left(n_{\lambda} \frac{\mathrm{d} x_{j}}{\mathrm{~d} l}\right)=\frac{\partial n}{\partial x_{j}}$,

with $j=1,2,3$. It can be rewritten as

$n_{\lambda} \frac{\mathrm{d}^{2} x_{j}}{\mathrm{~d} l^{2}}+\frac{\mathrm{d} n_{\lambda}}{\mathrm{d} l} \frac{\mathrm{d} x_{j}}{\mathrm{~d} l}=\frac{\partial n}{\partial x_{j}}$,

$\frac{\mathrm{d}^{2} x_{j}}{\mathrm{~d} l^{2}}=\frac{1}{n_{\lambda}}\left(\frac{\partial n_{\lambda}}{\partial x_{j}}-\frac{\mathrm{d} n_{\lambda}}{\mathrm{d} l} \frac{\mathrm{d} x_{j}}{\mathrm{~d} l}\right)$.

Changing the variable

$y_{j}=\frac{\mathrm{d} x_{j}}{\mathrm{~d} l}$

we obtain the following equation:

$y_{j}^{\prime}=\frac{1}{n_{\lambda}}\left(\frac{\partial n_{\lambda}}{\partial x_{j}}-\frac{\mathrm{d} n_{\lambda}}{\mathrm{d} l} y_{j}\right)$,

with

$\frac{\mathrm{d} n_{\lambda}}{\mathrm{d} l}=\frac{\mathrm{d} n_{\lambda}}{\mathrm{d} x_{j}} \frac{\mathrm{d} x_{j}}{\mathrm{~d} l}$.
From Eq. (A.4) we have

$x_{j}^{\prime}=y_{j}$.

Eqs. (A.5) and (A.7) define a system where $x_{j}$ represents the position and $y_{j}$ represents the velocity at a given point in the trajectory, which can be written in matrix form as

$\left(\begin{array}{l}x_{j} \\ y_{j}\end{array}\right)^{\prime}=\left(\begin{array}{c}y_{j} \\ \frac{1}{n_{\lambda}}\left(\frac{\partial n_{\lambda}}{\partial x_{j}}-\frac{\mathrm{d} n_{\lambda}}{\mathrm{d} l} y_{j}\right)\end{array}\right)$.

Eq. (A.8) has the form

$Y^{\prime}=f(l, Y)$

which defines an initial value problem with $Y(0)=\alpha$. The change of variable in Eq. (A.4) allows us to solve this problem by applying the embedded Runge-Kutta method RK5(4)7M from the Dormand-Prince family of methods. It has been chosen because it is an embedded method, which implies variable step and error control, and also because its convergence is much faster than other methods.

The RK5(4)7M obtains an approximate solution of $Y$ in several points of the interval $[A, B]$, and then interpolates for the rest of the points. All Runge-Kutta methods are characterized by a table of coefficients:

\begin{tabular}{l|llll}
$c_{1}$ & 0 & & & \\
$c_{2}$ & $a_{21}$ & $\ldots$ & 0 & \\
$\ldots$ & $\cdots$ & $\ldots$ & $\ldots$ & 0 \\
$c_{s}$ & $a_{s 1}$ & $\ldots$ & $a_{s s-1}$ & 0 \\
\hline & $b_{1}$ & $b_{2}$ & $\ldots$ & $b_{s}$ \\
& $\hat{b}_{1}$ & $\hat{b}_{2}$ & $\ldots$ & $\hat{b}_{s}$
\end{tabular}


whereas for the chosen RK5(4)7M method these coefficients are

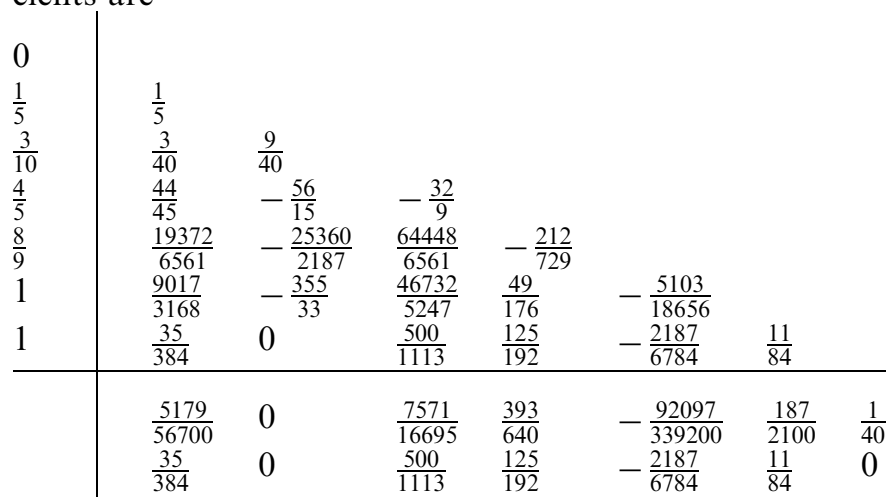

To obtain the different trial points $\left(x_{n}, y_{n}\right)$ which represent the approximation to the solution, we first need to obtain the $k_{n}^{i}$ coefficients from the following equation:

$k_{n}^{i}=f\left(x_{n}+h_{n} c_{i}, y_{n}+\sum_{j=1}^{i-1} a_{i j} k_{n}^{j}\right)$,

where $h_{n}$ is the step size in the iteration $n$ and $a_{i j} \mathrm{y} c_{i}$ are obtained from the table of coefficients. Given the coordinates $\left(x_{n}, y_{n}\right)$ of a point, the coordinates $\left(x_{n+1}, y_{n+1}\right)$ of the next point are obtained as

$x_{n+1}=x_{n}+h_{n}$,

$y_{n+1}=y_{n}+h_{n} \sum_{i=1}^{s} b_{i} k_{n}^{i}$,

where $b_{i}$ are given by the coefficients table and $k_{n}^{i}$ is obtained using Eq. (A.10). The other solution of the embedded pair is obtained as

$\hat{y}_{n+1}=y_{n}+h_{n} \sum_{i=1}^{s} \hat{b}_{i} k_{n}^{i}$

The error estimation $E_{n}$ for each step is then computed as $E_{n}=y_{n}-\hat{y}_{n}$.

If this error is greater than a given tolerance tol, the step is modified and the solutions recalculated. If the error is less than such tolerance, the next point is calculated while also varying the step. The new step is calculated as follows

$h_{n+1}=h_{n}\left(\frac{t o l}{\left\|E_{n}\right\|}\right)^{(1 / p)}$.

Given the numerical precision used and the round error when implementing the method, it could be that the error $\left\|E_{n}\right\|$ becomes zero; values of $\left\|E_{n}\right\|$ less than a given $\varepsilon$ must therefore be treated differently. In this implementation we chose to multiply the step times ten when this happens.

\section{References}

[1] Preetham AJ, Shirley P, Smits BE. A practical analytic model for daylight. In: Rockwood A, editor. Proceedings of the conference on computer graphics (SIGGRAPH99). New York: ACM Press; 1999. p. 91-100.

[2] Dobashi Y, Nishita T, Kaneda K, Yamashita H. A fast display method of sky colour using basis functions. In: Thalmann NM, Thalmann D, Shin SY, Kunii TL, Kim M-S, editors. The journal of visualization and computer animation, vol. 8(2). New York: Wiley; 1997. p. 115-27.

[3] Jensen HW, Durand F, Stark MM, Premoze S, Dorsey J, Shirley P. A physically-based night sky model. In: Fiume E, editor. SIGGRAPH 2001, computer graphics proceedings, annual conference series. ACM Press/ACM SIGGRAPH; 2001. p. 399-408.

[4] Nishita T, Sirai T, Tadamura K, Nakamae E. Display of the earth taking into account atmospheric scattering. In: Kajiya JT, editor. Computer graphics (SIGGRAPH '93 proceedings), vol. 27; 1993. p. $175-82$.

[5] Irwin J. Full-spectral rendering of the earth's atmosphere using a physical model of Rayleigh scattering. In: Proceedings of the 14th annual eurographics UK conference, vol. 1; 1996. p. 103-15.

[6] Jackèl D, Walter B. Modeling and rendering of the atmosphere using mie-scattering. Computer Graphics Forum 1997;16(4):201-10.

[7] Riley K, Ebert DS, Kraus M, Tessendorf J, Hansen C. Efficient rendering of atmospheric phenomena. In: Fellner D, Spencer S, editors. Proceedings of the 2004 Eurographics Symposium on Rendering. p. 375-86.

[8] Dobashi Y, Yamamoto $\mathrm{T}$, Nishita $\mathrm{T}$. Interactive rendering of atmospheric scattering effects using graphics hardware. In: SIGGRAPH/eurographics workshop on graphics hardware. Eurographics Association; 2002. p. 99-107.

[9] Sloup J. A survey of the modelling and rendering of the earth's atmosphere. In: SCCG '02: Proceedings of the 18th spring conference on computer graphics. New York, NY, USA: ACM Press; 2002. p. $141-50$.

[10] Berger M, Trout T, Levit N. Ray tracing mirages. IEEE Computer Graphics and Applications 1990;10(3):36-41.

[11] Musgrave FK. A note on ray tracing mirages. IEEE Computer Graphics and Applications 1990;10(6):10-2.

[12] Lehn WH. A simple parabolic model for optics of the atmospheric surface layer. In: Applied mathematical modelling, vol. 9; 1985. p. $447-53$.

[13] Linţu A, Haber J, Magnor M. Realistic solar disc rendering. In: Skala V, editor. WSCG'2005 full papers conference proceedings; 2005. p. 79-86.

[14] Seron F, Gutierrez D, Gutierrez G, Cerezo E. Implementation of a method of curved ray tracing for inhomogeneous atmospheres. Computers \& Graphics 2005;29:95-108.

[15] Wyszecki G, Stiles WS. Color science: concepts and methods. Quantitative data and formulae. New York: Wiley; 1982.

[16] Glassner AS. Principles of digital image synthesis. San Francisco, CA, USA: Morgan Kaufmann; 1995.

[17] Born M, Wolf E. Principles of optics: electromagnetic theory of propagation, interference and diffraction of light. Cambridge: Cambridge University Press; 2002.

[18] Burden RL, Faires J. Numerical analysis, 4th ed. Boston: PWS-Kent; 1988.

[19] Dormand J, Prince P. A family of embeded Runge-Kutta formulae. J. Computational and Applied Mathematics 1980;6(1):19-26.

[20] USGPC, US Standard Atmosphere. United State Government Printing Office, Washington, DC; 1976.

[21] Young A. Sunset science iii. Visual adaptation and green flashes. Journal of the Optical Society of America 2000;A(17):2129-39.

[22] Gladstone J, Dale J. On the influence of temperature on the refraction of light. Philosophical Transactions 1858;148:887.

[23] Ciddor PE. Refractive index of air: new equations for the visible and near infrared. Applied Optics 1996;35:1566-73.

[24] van der Werf SY. Ray tracing and refraction in the modified US1976 atmosphere. Applied Optics 2003;42(3):354-66.

[25] van der Werf SY, Können GP, Lehn WH. Novaya Zemlya effect and sunsets. Applied Optics 2003;42(3):367-78. 
[26] The star almanac for land surveyors, 1st ed. Her Majesty's Nautical Almanac Office, London: 1951.

[27] The nautical almanac, 1st ed. Her Majesty's Nautical Almanac Office, London, and Nautical Almanac Office of the United States, Washington, DC; 1958.

[28] B.V.K. Refraction tables of the Pulkovo Observatory, 5th ed. Nauka: Leningrad; 1985.

[29] Stam J, Languenou E. Ray tracing in non-constant media. In: Proceedings of the eurographics workshop on rendering techniques '96. London, UK: Springer; 1996. p. 225.

[30] Owens JC. Optical refractive index of air: dependence on pressure, temperature and composition. Applied Optics 1967;6:51-9.

[31] Minnaert M. Light and colour in the outdoors. New York: Springer; 1993.

[32] Malaurie A. Ultima Thule, explorers and natives in the Polar North. W W Norton \& Co Ltd, December 2003.

[33] 〈http://www.islandnet.com/see/weather/history/artmirge.htm $\rangle$, December 2005.

[34] Sawatzky HL, Lehn WH. The Arctic mirage and the early North Atlantic. Science Magazine 1976;192:1300-5.
[35] Tolkien JRR, Gordon EV, editors. Sir Gawain and the Green Knight, 2nd edition by Norman Davis. Oxford: Clarendon Press; 1967, ISBN 0198114869.

[36] Zeeberg JJ. Climate and glacial history of the Novaya Zemlya Archipelago, Russian Arctic: with notes on the Region's history of exploration. The Netherlands: Rozenberg Publishers; 1 April 2002.

[37] 〈http://mintaka.sdsu.edu/gf//, September 2005.

[38] Verne J. In: Hetzel J, editor. Le Rayon-Vert. Paris, 1882.

[39] Gutierrez D, Anson O, Munoz A, Seron F. Perception-based rendering: eyes wide bleached. In: Dingliana J, Ganovelli F, editors. EG '05: short papers proceedings of the Eurographics 2005. The Eurographics Association and the Image Synthesis Group; 2005. p. 49-52.

[40] Bregler C, Cohen M, Debevec P, McMillan L, Sillion F, Szeliski R. Image-based modeling, rendering, and lighting. SIGGRAPH 99 Course 39.

[41] Greenler R. Rainbows, Halos and Glories. Cambridge: Cambridge University Press; 1990. 